\title{
Transition to collective oscillations in finite Kuramoto ensembles
}

\author{
Franziska Peter ${ }^{1}$ and Arkady Pikovsky ${ }^{1,2}$ \\ ${ }^{1}$ Institute of Physics and Astronomy, University of Potsdam, \\ Karl-Liebknecht-Straße 24-25, 14476 Potsdam, Germany \\ ${ }^{2}$ Research Institute for Supercomputing, Nizhny Novgorod State University, \\ Gagarin Av. 23, 606950, Nizhny Novgorod, Russia
}

(Published: 20 March 2018)

\begin{abstract}
We present an alternative approach to finite-size effects around the synchronization transition in the standard Kuramoto model. Our main focus lies on the conditions under which a collective oscillatory mode is well defined. For this purpose, the minimal value of the amplitude of the complex Kuramoto order parameter appears as a proper indicator. The dependence of this minimum on coupling strength varies due to sampling variations and correlates with the sample kurtosis of the natural frequency distribution. The skewness of the frequency sample determines the frequency of the resulting collective mode. The effects of kurtosis and skewness hold in the thermodynamic limit of infinite ensembles. We prove this by integrating a self-consistency equation for the complex Kuramoto order parameter for two families of distributions with controlled kurtosis and skewness, respectively.
\end{abstract}

\section{INTRODUCTION}

Synchronization in ensembles of self-sustained oscillators is a universal phenomenon, relevant not only for many physical and technical applications (e.g., laser arrays [1], electrochemical oscillators [2], and power grids [3]) but also for the self-perpetuation of living beings. In such biological systems, the manifestations of synchrony are often spectacular-like the united rhythmic flashing of mating fireflies that attracts tourists over vast distances or synchronized brain waves that apparently speed up learning [4]. From single-cell organisms to animals through to humans, many species benefit from synchronizing their motions, metabolism, cell division, gene expression, circadian cycles and many other inner rhythms.

Arthur Taylor Winfree's model of large ensembles of self-sustained oscillatory systems [5] and its modification to an analytically solvable mean-field system by Yoshiki Kuramoto [6, 7] established the understanding of synchronization as a nonequilibrium phase transition. Since then, various extensions to this Kuramoto model contributed to a broader, more realistic, picture of synchronization dynamics in large ensembles: the generalization to higher modes of the coupling function [8, 9], the introduction of a phase shift [10] to the coupling function, the addition (or multiplication) of noise [11], and the investigation of different coupling network structures 12]. Two significant analytical achievements allow for a deeper understanding of the phase space geometry: the WatanabeStrogatz reduction for finite ensembles of identical oscillators 13] and the Ott-Antonsen ansatz for infinite ensembles of distributed oscillators 14].

Most of the present theoretical approaches to synchronization concentrate on infinite populations, while the theory on finite-size ensembles evolves only gradually. However, only a few experiments study really large numbers (thousands) of oscillatory units [1, 15], while experimental setups of populations up to a hundred oscillators dominate the area 2, 16, 17]. Many numerical and theoretical studies focus on the properties of either rather small systems-e.g., Ref. 18] explores the chaotic dynamics in a Kuramoto model with only 4 to 20 oscillators-or much larger ensembles of $10^{4}$ or more oscillators, where most efforts are dedicated to the scaling properties of fluctuations of the order parameter 19 23].

In this paper we explore ensembles of moderate size (typically 50 to 200 oscillators)-corresponding to realistic experimental conditions. In this respect, this study contributes to closing the gap between the two extremes of relatively small and very large ensembles by investigating effects that naturally emerge in small ensembles and extend to the infinite limit depending on the natural frequency distribution.

Our approach to the finite-size problem differs from the scaling-of-fluctuations approach adopted in [19 23]. We dedicate the primary focus to the question: Under which conditions is a collective mode well defined in a finite ensemble of coupled phase oscillators? In the thermodynamic limit, the exact dynamical equations for the complex order parameter can be derived in some cases. The most prominent example is the Ott-Antonsen theory for the Kuramoto-Sakaguchi system [14]. In other cases, at least an asymptotic solution for infinitely large ensembles can be interpreted as oscillations of a collective mode. In general, the main feature that distinguishes self-sustained oscillations from the noise-driven ones is the existence of a macroscopic phase: It is well defined for self-sustained oscillations, but ill defined for noisy states where the amplitude can vanish.

In finite ensembles of Kuramoto type-in contrast to the thermodynamic limit-the complex order parameter fluctuates strongly. It is suggestive to consider a collective mode as a well-defined macroscopic oscillation, if the corresponding macroscopic phase is well-defined for all times. This means that the amplitude should not vanish (cf. Ref. 17] where this idea applies to experimental studies of a finite set of oscillators). Below, we study in 
detail the statistical properties of the minimum of the amplitude of the complex order parameter that serves as an indicator for the emergence of a global oscillatory mode. These properties strongly depend on the particular sample of frequencies. The observed effects can be traced in the thermodynamic limit by exploring distributions with controlled kurtosis and skewness.

The paper is organized as follows: In Sec. III we introduce the model and discuss the phenomenology of the complex order parameter dynamics for both infinite and finite ensembles. Section III introduces the minimum of the amplitude of the order parameter as an indicator for the presence of a collective mode. Section IV discusses the effect of sample kurtosis and sample skewness of the natural frequency distribution on the synchronization transition characterized by the introduced indicator and on the global phase dynamics, respectively. These properties persist in the thermodynamic limit, as we prove in Sec. $\nabla$ for distribution families with parameters for kurtosis and skewness, respectively. Section VI contains conclusion and outlook.

\section{SYNCHRONIZATION TRANSITION: THERMODYNAMIC LIMIT VS. FINITE SYSTEM}

In this section, we introduce the Kuramoto model of coupled phase oscillators and compare the dynamics of the complex order parameter in infinite and finite ensembles. We discuss the role of coupling strength and natural frequencies in both cases.

The standard Kuramoto model describes $N$ nearly identical phase oscillators with weak sinusoidal coupling. Their natural frequencies $\omega_{i}$ spread according to some distribution $g(\omega)$. Phases $\theta_{i}$ are globally coupled with strength $\epsilon$,

$$
\dot{\theta}_{i}=\omega_{i}+\frac{\epsilon}{N} \sum_{j=1}^{N} \sin \left(\theta_{j}-\theta_{i}\right)=\omega_{i}+\epsilon R \sin \left(\varphi-\theta_{i}\right),
$$

via a complex mean field $Z$, defined as

$$
Z=R \mathrm{e}^{\imath \varphi}=\frac{1}{N} \sum_{j=1}^{N} \mathrm{e}^{\imath \theta_{j}} .
$$

The absolute value of $Z$, called Kuramoto order parameter $R$, quantifies the degree of phase coherence in the population and thereby serves as an indicator for synchrony.

Shifting all frequencies by a constant $\omega_{i} \rightarrow \omega_{i}+\Delta \omega$ is equivalent to transforming to a rotating reference frame with frequency $\Delta \omega$ to the entire system. This rotational invariance proves beneficial in the thermodynamic limit, where the complex mean field rotates uniformly. This rotation becomes stationary in an appropriate reference frame, which us allows us to describe a synchronous state as a steady one. Similarly, scaling all frequencies by a constant factor $\omega_{i} \rightarrow \sigma \omega_{i}$ just scales time and the coupling strength by the same factor. Thus, without loss of generality, we set the standard deviation of $g(\omega)$ to 1 and shift the mean frequency to zero in all examples and all numerical experiments (in the latter case: after sampling).

\section{A. Solution of the Kuramoto model in the thermodynamic limit}

In the thermodynamic limit of infinite ensembles, $N \rightarrow$ $\infty$, the dynamics of the complex mean field $Z$ as a function of coupling strength $\epsilon$ demonstrates a transition to synchrony - comparable to a nonequilibrium phase transition [6, 7]. In this section we recall a basic qualitative picture of this transition for symmetric unimodal distributions (thus assuming their maximum at the mean frequency $\bar{\omega}$ ) and describe a quantitative method for finding the order parameter as a function of $\epsilon$.

States $\left\{\theta_{i}\right\}$ with vanishing order parameter are always solutions, irrespective of the coupling strength. With $R=0$, the oscillators perfectly decouple and rotate with their respective natural frequencies. Therefore, the individual phases are fully incoherent, i.e., uniformly distributed in $[0,2 \pi)$, so that $R$ vanishes exactly, reflecting the self-consistent nature of the problem. Another, nontrivial, solution with $R>0$ exists above the critical coupling $\epsilon_{c}^{\infty}=2 \cdot[\pi g(\bar{\omega})]^{-1}$. The order parameter $R$ as a function of coupling strength $\epsilon$ is typically continuous but not differentiable in $\epsilon_{c}^{\infty}$. Only distributions with a symmetric plateau around $\bar{\omega}$ produce a jump at the critical coupling strength 24], with the uniform distribution as a special case 25]. (Multimodal distributions typically exhibit hysteresis [26].)

Coupling strengths above $\epsilon_{c}^{\infty}$ may lock only a fraction of the oscillators to a common frequency-except, e.g., in the case of a uniform frequency distribution, where the oscillators jump from zero to full frequency locking at $\epsilon_{c}^{\infty}$. The fraction of frequency locked oscillators increases with coupling strength, reflected in a growing order parameter. For distributions with compact support, the maximal frequency difference determines a coupling strength above which all oscillators rotate with the same observed frequency. As long as $\epsilon$ is finite, they maintain finite phase differences, and the order parameter asymptotically approaches $R=1$. For distributions with unbounded support, the fraction of asynchronous oscillators is always finite.

Quantitatively, the Kuramoto problem in the thermodynamic limit can be solved as follows 27 29]. One seeks for a solution that is stationary (in the sense of a stationary distribution function of phases, see Ref. [14]) in a frame rotating with some frequency $\Omega$, i.e., $\varphi=\Omega t+\varphi_{0}$, where $\varphi_{0}$ is a constant. The relative phase $\psi=\theta-\Omega t-\varphi_{0}$ obeys

$$
\dot{\psi}=\omega-\Omega-\epsilon R \sin (\psi), \quad R=\left\langle e^{i \psi}\right\rangle .
$$


It is convenient to consider $\Omega$ and $a=\epsilon R$ as parameters in Eq. (3). The distribution of phases $\psi$ at given $\omega$ can then be expressed as $\delta(\psi-\arcsin [(\omega-\Omega) / a)]$ for synchronous and $\sqrt{(\omega-\Omega)^{2}-a^{2}}|\omega-\Omega-a \sin \psi|^{-1}$ for asynchronous oscillators, respectively. The definition of the mean field $R$ can be expressed as a complex function of $\Omega$ and $a$, consisting of one real and two complex integrals (the other real integral, stemming from the asynchronous oscillators combined with the cosine, vanishes due to $2 \pi$ periodicity):

$$
\begin{gathered}
R=F(\Omega, a)=a \int_{-\pi / 2}^{\pi / 2} \mathrm{e}^{i \theta} g(\Omega+a \sin \theta) \cos \theta \mathrm{d} \theta \\
+\frac{i}{2 \pi} \int_{-\pi}^{\pi} \sin \theta \int_{|\omega-\Omega|>a} g(\omega) \frac{\sqrt{(\omega-\Omega)^{2}-a^{2}}}{|\omega-\Omega-a \sin \theta|} \mathrm{d} \omega \mathrm{d} \theta .
\end{gathered}
$$

In practice, both analytical and numerical integration of this equation provide a solution in parametric form with parameter $a$. First, as $R$ is a real quantity, the imaginary part of the right-hand side must vanish. This condition assigns a unique value $\Omega_{a}$ to frequency $\Omega$ for given $a$. For symmetric distributions, the imaginary integral vanishes and $\Omega=0$. The remaining real integral $F\left(\Omega_{a}, a\right)$ provides the dependence of order parameter $R$ on coupling strength $\epsilon$, namely $R=F\left(\Omega_{a}, a\right)$ and $\epsilon=a / F\left(\Omega_{a}, a\right)$.

For some special frequency distributions $g(\omega)$, the integrals in Eq. (41) can be calculated analytically. The simplest case of identical oscillators [such that $g(\omega)$ degenerates to a delta distribution] has only one nontrivial solution, $R=1$. For a Gaussian distribution $g(\omega)=\mathrm{e}^{-\omega^{2} / 2} /(\sqrt{2 \pi})$, the parametric solution can be expressed via the modified Bessel functions of the first kind $\mathcal{I}_{\mu}[30]$ :

$$
R=\sqrt{\pi A / 2} \mathrm{e}^{-A}\left[\mathcal{I}_{0}(A)+\mathcal{I}_{1}(A)\right],
$$

where $A=a^{2} / 4$. Similarly, the solution for the Laplace distribution $g(\omega)=\mathrm{e}^{-\sqrt{2}|x|} /(\sqrt{2})$ includes the modified Bessel functions of the first kind $\mathcal{I}_{\mu}$ and the modified Struve functions $\mathcal{L}_{\mu}$ :

$$
R=\pi \cdot\left[\mathcal{I}_{1}(B)-\mathcal{L}_{1}(B)\right] / 2,
$$

where $B=a \sqrt{2}$.

For uniform distributions with mean zero, height $h$, and width $2 \omega_{\max }$ (with normalization $2 \omega_{\max } \cdot h=1$ ), solutions with $\omega_{\max } / a \geq 1$ correspond to $\epsilon_{c}^{\infty}=2 /(\pi h)$ with $R$ in $(0, \pi / 4]$. Solutions with $\omega_{\max } / a<1$ obey the parametric equation

$$
R=2 a \omega_{\max } \arcsin \left(\frac{\omega_{\max }}{a}\right)+\frac{1}{2} \sqrt{1-\left(\frac{\omega_{\max }}{a}\right)^{2}} .
$$

Due to Eq. (11), oscillators with $\left|\omega_{i}\right|<\epsilon R$ have a stable fixed point (i.e., lock to the frequency of the global phase), which is true for any coupling stronger than $\epsilon_{c}^{\infty}=2 /(\pi h)$, where $R>\pi / 4$, such that $\epsilon R>1 /(2 h)=$ $\omega_{\max } \geq\left|\omega_{i}\right|$. This means that at the critical coupling strength all oscillators jump to full synchrony in the sense of full frequency locking.

A number of other distributions can be integrated by the same method as well, for instance, the distributions listed in Ref. [25].

\section{B. Transition and phenomenology in finite populations}

In a finite population, the solutions described above are not exact, most obviously evident in the fluctuations of the order parameters $Z$ and $R$. These fluctuations start from a finite value $\sim 1 / \sqrt{N}$ for vanishing coupling, because states with $R=0$ are not invariant in the finite model. Fluctuations increase around the critical coupling and then decay for stronger coupling. Thus, the transition to synchrony becomes blurry when measured by means of the averaged order parameter. The details of the dynamics of the order parameters strongly depend on the way the finite frequency sample is generated from the underlying distribution $g(\omega)$. Here, either regular or random sampling may be implemented, depending on the specific question. Regular sampling by virtue of the quantiles of the distribution (i.e., inverse transform sampling from equidistant points) allows for a straightforward comparison between different ensemble sizes, as in this case the frequencies in each sample are uniquely determined by the system size $N$. Random sampling (i.e., sampling with some random number generator) results in a finite sample-to-sample variability which decreases as $N$ grows. This variability of samples complicates comparisons, as a statistical analysis becomes necessary. Yet, it is the method of choice to fully represent the finite-size effects of the underlying distribution in an unbiased fashion.

Figure 1 1 shows a typical time evolution of the complex order parameter in an ensemble of 50 oscillators for three samples of $g(\omega)$. In the two left panels, a normal distribution $\mathcal{N}(0,1)$ is sampled (a) randomly and (b) regularly. Figure $1(\mathrm{c})$ shows $Z(t)$ for a random sample of a Lorentz distribution which is widely used in studies on the $\mathrm{Ku}$ ramoto model because of analytic tractability in the infinite limit. In all three cases, the order parameter $Z$ fluctuates around zero for small coupling strengths, corresponding to predominantly asynchronous motion below the synchronization transition. For stronger coupling $\epsilon$, the complex order parameter $Z=R e^{i \varphi}$ is clearly separated from zero. For moderate coupling strengths and regular sampling, only the amplitude $R$ performs sustained fluctuations, while the argument $\varphi$ converges to a constant (see Secs. IVB and VB for an explanation). In contrast, random samples exhibit sustained fluctuations in both $R$ and $\varphi$, for the same moderate coupling strengths. In all cases, $Z$ quickly converges to a complex 


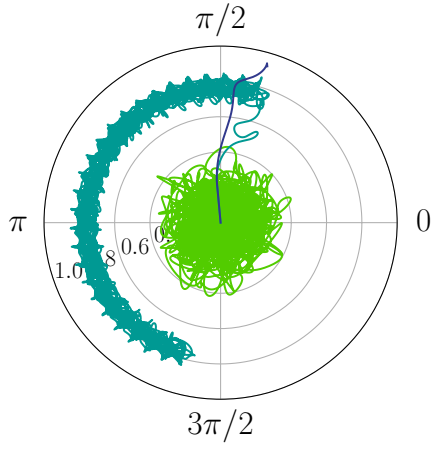

(a)

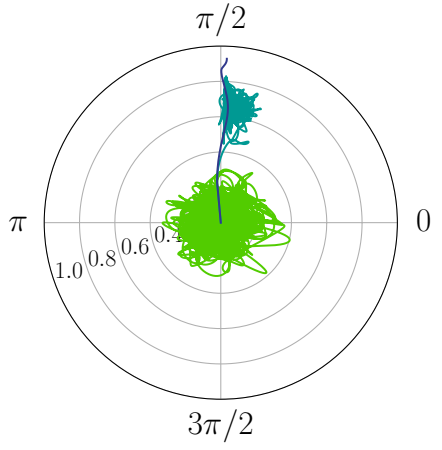

(b)

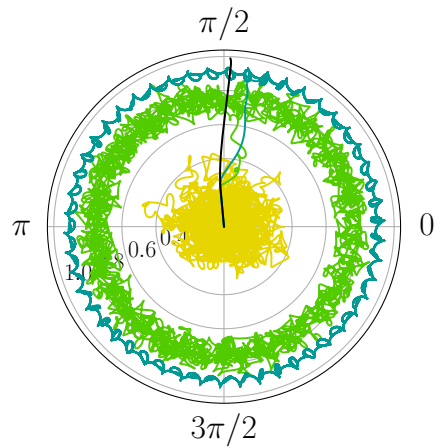

(c) coupling strengths

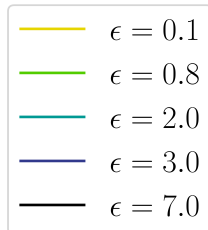

central moments

\begin{tabular}{ccccc}
$\mu$ & $\sigma^{2}$ & $\gamma_{1}$ & $\beta_{2}$ \\
\hline (a) & 0 & 1 & 0.07 & 3.8
\end{tabular}

(b) $0 \begin{array}{llll}0 & 1 & 0.00 & 2.5\end{array}$

$\begin{array}{llllll}\text { (c) } & 0 & 1 & 1.80 & 16.1\end{array}$

FIG. 1. (Color online) Polar representation of the time evolution $\left(t=10^{3}\right)$ of the complex order parameter $Z$ at different values of coupling strengths $\epsilon$ for 50 oscillators with frequencies: (a) randomly sampled from a normal distribution, (b) regularly sampled via quantiles from a normal distribution, and (c) randomly sampled from the Lorentz distribution. Note that in panels (a) and (b) we plot only $\epsilon=0.8,2.0$, and 3.0, while in (c) we show $\epsilon=0.1,0.8,2.0$, and 7.0 for better clarity. The table shows the first sample moments of the three natural frequency samples: mean $\mu$, variance $\sigma^{2}$, skewness $\gamma_{1}$, and kurtosis $\beta_{2}$. All trajectories start from the same initial phases, randomly picked from a uniform distribution in $[0,2 \pi)$, such that $R\left(t_{0}\right) \approx 0$. All numerical time evolutions in this paper use the fourth-order Runge-Kutta scheme with step size 0.01 .

constant for sufficiently strong coupling. The coupling strength necessary to achieve this state is considerably higher $(\epsilon \sim 7)$ for the Lorentz distribution sample than for the two Gaussian samples $(\epsilon \sim 3)$.

Most of the previous works on finite-size effects in the Kuramoto transition [19, 20, 23, 31, 32] focus on the statistics of fluctuations of the real order parameter $R$. Let us here focus on the phase of the complex mean field $\varphi$. This is important if we want to interpret the dynamics of the complex mean field $Z$ as that of a complex amplitude of an effective collective oscillatory mode. As mentioned briefly in the Introduction, such interpretation is mathematically justified for the Kuramoto model in the thermodynamic limit with Lorentz-distributed frequencies. In this case, the Ott-Antonsen ansatz 14 reduces the dynamics to a Stuart-Landau-type equation for the order parameter $Z$. In other cases, such a reduction is justified at least close to the transition point [33, 34]. From this macroscopic viewpoint, the transition to synchrony in a population corresponds to a Hopf bifurcation from a fixed point to stable self-sustained oscillations of the mean field. The dynamics of the macroscopic phase $\varphi$ displays the temporal coherence of these oscillations.

In all three cases depicted in Fig. 1, the order parameter strays around zero for small coupling strengths but avoids an inner circle for stronger coupling. This implies a problem in the definition of a macroscopic phase $\varphi$ for weak coupling: If amplitude $R$ vanishes, then the phase of oscillations is ill defined. This suggests to distinguish the two domains of the dynamics of the complex mean field, according to the minimal possible value of the amplitude $R_{\min }$ (see Ref. [17], where this parameter has been applied to the analysis of experiments with a finite set of coupled oscillators):
- If $R_{\min }=0$, then the macroscopic phase $\varphi$ is not defined globally, and thus macroscopic oscillations are ill defined. The complex order parameter diffuses around zero.

- If $R_{\min }>0$, then the macroscopic phase $\varphi$ is well defined. It validates the term "macroscopic oscillations" and defines their coherence. The complex order parameter is well separated from zero.

In the following section, we focus on the properties of $R_{\text {min }}$.

\section{MINIMAL VALUE OF THE ORDER PARAMETER AS AN INDICATOR FOR THE TRANSITION TO A COLLECTIVE MODE}

In the preceding section, we argued that $R_{\min }$ is an appropriate quantity to characterize the emergence of collective oscillations in a finite population. In this section, we discuss in detail the statistical properties of this parameter. In Fig. 2 we show the dependence of $R_{\text {min }}$, calculated over a time interval $0 \leq t \leq 10^{5}$, on the coupling parameter $\epsilon$ for one random sample of a Gaussian distribution of frequencies. In contrast to time-averaged value $\langle R\rangle_{t}$ which smoothly depends on $\epsilon, R_{\text {min }}$ undergoes a sharp transition at $\epsilon_{c}^{\min } \approx 1.82$.

From a statistical point of view, the calculation of $R_{\text {min }}$ is less stable than that of the averaged value, as it is dominated by the tail of the distribution of $R$. This is illustrated in Fig. 2(b), where we show $R_{\min }$ as a function of observation time. For strong coupling, $R_{\text {min }}$ saturates already at about $t \approx 10^{3}$. By contrast, at weak or even vanishing coupling, where the oscillators effectively decouple, $R_{\min }$ has no lower bound. Due to their 

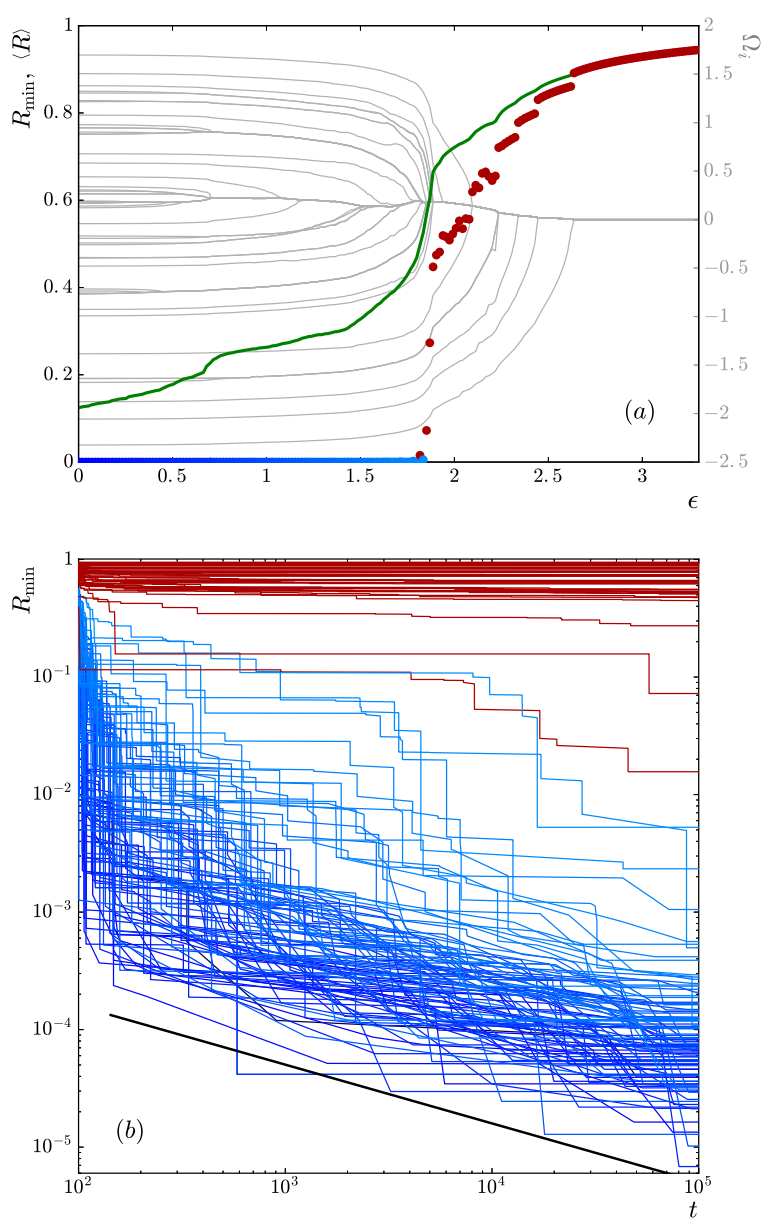

FIG. 2. (color online) Statistical characterization of the minimum of $R(t)$, for $N=50$ oscillators and a fixed random sample of a Gaussian frequency distribution with sample moments $\mu=0, \sigma^{2}=1, \gamma_{1}=-0.45, \gamma_{2}=-0.55$. Time evolution starts from uniformly distributed phases in $[0,2 \pi)$. (a) $R_{\min },\langle R\rangle$, and $\Omega_{i}$ vs. coupling strength $\epsilon$. Here $R_{\min }$ is shown vs. $\epsilon$ at $t=10^{5}$ (after transients of length $10^{2}$ ) for an $\epsilon$ grid with $\Delta \epsilon \approx 0.02$. The green bold solid line shows the mean value $\langle R\rangle_{T}$ averaged over a time interval of $T=10^{4}$. The observed individual frequencies $\Omega_{i}$ shown in gray (right-hand scale) reveal which oscillators synchronize already at small frequencies and which join the synchronous cluster only at stronger coupling. The sampled natural frequencies $\omega_{i}$ equal the observed frequencies $\Omega_{i}$ at zero coupling. (b) Dependence of $R_{\min }$ on the observation time. The bold black line gives an estimate $t^{-1 / 2}$ for the scaling behavior. In both panels, red indicates $R_{\min }>0.01$ at $t=10^{5}$, while we color the remaining sub-critical trajectories in blue.

different frequencies, a vicinity of any configuration of phases is visited and that vicinity shrinks with growing length of the time series. Thereby, arbitrary small values of $R_{\text {min }}$ become increasingly probable with longer observation time. The finite-time observation roughly follows the law $R_{\min } \sim t^{-1 / 2}$ (black bold line), which compares to a random sampling of a two-dimensional distribution of $R$ with finite density at zero.

The numeric evaluation of $R_{\min }$ is most unreliable near the critical point, where the decrease of $R_{\min }(t)$ with observation time $t$ is extremely slow (one can see several such realizations in Fig. 21). This appears unavoidable, because the time scale typically diverges at the criticality, see, e.g., Ref. [35]. Nevertheless, the sharp transition in the dependence of $R_{\min }$ on $\epsilon$ is well pronounced and reliable for calculations, see Fig. 2(a).

Beyond transition, $R_{\min }(\epsilon)$ follows a curve that is generally growing, but not everywhere monotonous. At even stronger coupling, several seemingly quite regular jumps dominate the picture. To understand this, a juxtaposition of $R_{\min }(\epsilon)$ and the individual observed frequencies $\Omega_{i}$ of the oscillators (in gray) is quite instructive: The jumps correspond to events where oscillators join the major synchronous cluster, built by oscillators with similar natural frequencies. At these jumps, the number of incommensurate contributions to $Z$ decreases and the dynamics of $Z(t)$ becomes more ordered, and eventually periodic when all oscillators have joined the synchronized cluster.

Remarkably, the oscillators' observed frequencies reveal frequency-locked clusters far below the critical coupling. The vast majority of oscillators, however, joins the central synchronized cluster at about the critical coupling strength.

In Fig. 2, we present the dependence of $R_{\min }$ on coupling strength and observation time for just one random sample of a Gaussian distribution [see Fig. 22(a) at $\epsilon=0$ ]. The next section discusses the sampling variation of the observed effects and the scaling with ensemble size $N$.

\section{EFFECTS OF KURTOSIS AND SKEWNESS IN FINITE ENSEMBLES}

The moments of a finite random sample are random variables, distribution of which depends on $N$ and on the underlying distribution. For instance, the mean of a Gaussian sample of size $N$ of a Gaussian with variance $\sigma^{2}$ is itself Gaussian distributed with variance $\sigma^{2} / N$. In Eq. (1), changing mean and variance of $g(\omega)$ merely corresponds to shifting to a different rotating reference frame and a different coupling parameter range, respectively. The deviations of the next higher moments, skewness and kurtosis, in contrast, cannot be rescaled and potentially determine properties of the transition and the dynamics in finite ensembles. In this section, we investigate the effect of sample skewness and sample kurtosis of a Gaussian natural frequency distribution.

\section{A. Sample kurtosis of $g(\omega)$ determines the shape of the transition curve $R_{\min }(\epsilon)$}

In this section, we identify the sample kurtosis as the main reason for the spread of the $R_{\min }(\epsilon)$ curves, in par- 

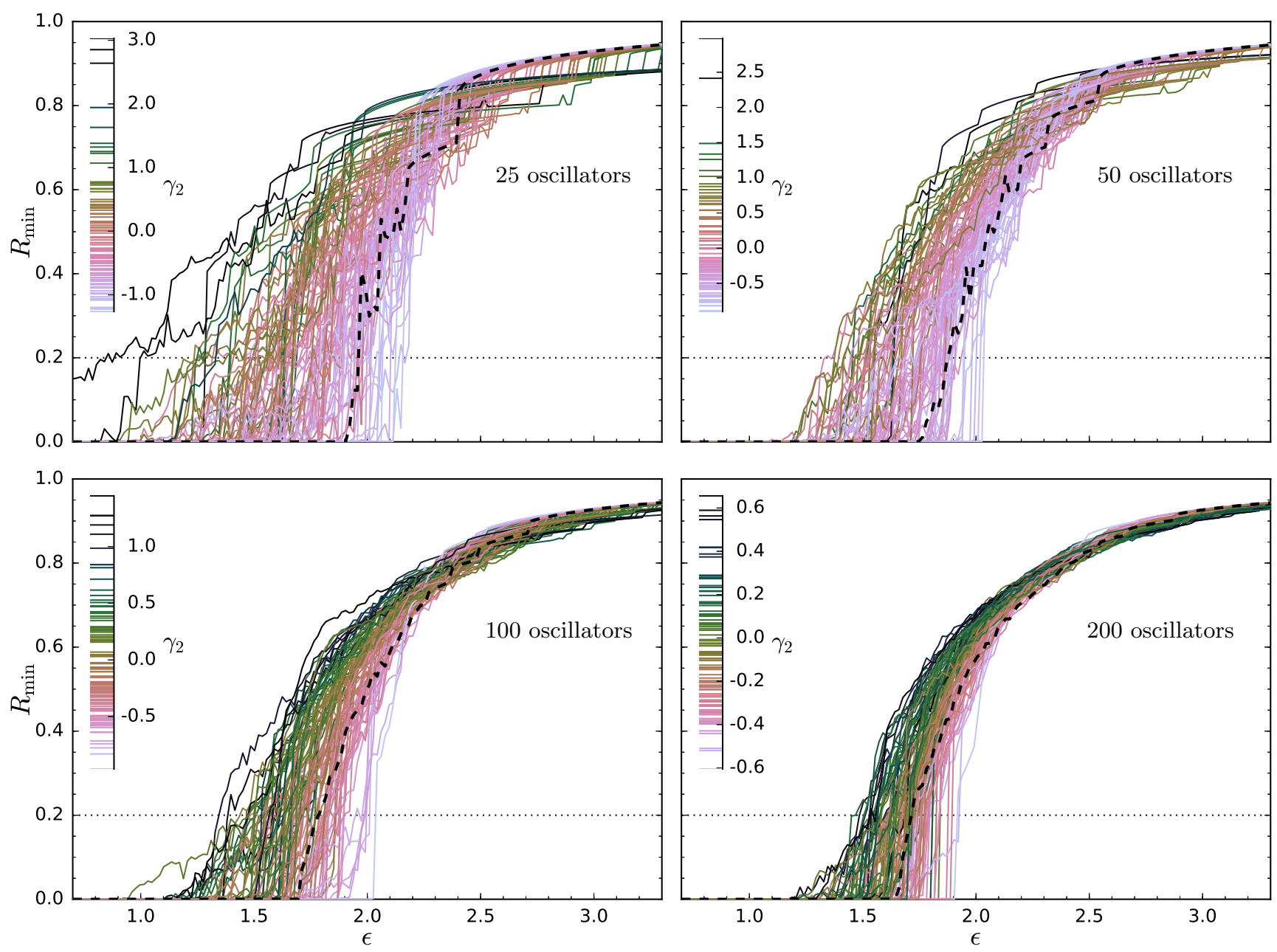

FIG. 3. (color online) Dependence of $R_{\min }$ on $\epsilon$ : Each panel shows results for 100 random samples of a Gaussian frequency distribution for $N=25,50,100,200$. The curves are gray shaded (colored in the online version) according to their respective excess kurtosis $\gamma_{2}$. Note that the range of $\gamma_{2}$ differs considerably between the panels. The black dashed line stems from regular sampling. The dotted black line marks the cut at $R_{\min }=0.2$, at which the spread presented in Fig. 4 is measured.

ticular for the spread of the critical values of coupling at which $R_{\min }$ becomes nonzero. Furthermore, we quantify the scaling of this spread with ensemble size.

Figure 3 presents the results of a numerical experiment, in which we generate 100 frequency samples of a Gaussian distribution $\mathcal{N}(0,1)$ for each of the ensemble sizes $N=25,50,100,200$. For 150 coupling strengths $\epsilon$ ranging from 0.7 to 3.3 and for each frequency sample, we initiate a time series from one fixed set of uniformly distributed random phases. We plot the minimum of $R$ after observation time $t=10^{5}$ (with initial transients of length $t=10^{4}$ ) versus coupling strength $\epsilon$. Each line in the plot is gray shaded (colored in the online version) according to the sample kurtosis of the respective frequency sample.

Kurtosis-the fourth standardized central moment $\beta_{2}=$ $\left\langle\omega_{i}^{4}\right\rangle\left\langle\omega_{i}^{2}\right\rangle^{-2}$-quantifies the probability weight in the tails of a probability distribution. The kurtosis of a Gaus- sian equals 3 (mesokurtic distribution), therefore comparisons among different distributions often refer to excess kurtosis $\gamma_{2}=\beta_{2}-3$. Positive excess kurtosis (leptokurtic distribution) often indicates fatter tails-which in our case means many moderately extreme frequencies that require stronger coupling to join the synchronization cluster. Negative excess kurtosis (platykurtic distribution) corresponds to distributions with more probability weight concentrated closely around the mean [36]-here we have a broader range of small frequencies with an almost constant probability and few but extreme outliers 37]. This can be understood as follows: Under the restriction of unit standard deviation, the few outliers in platikurtic samples must be significantly larger in their absolute value than the many outliers in the fat tails of leptokurtic samples, because they must compensate for the tightly packed frequencies gathered around the mean to give the same standard deviation. 


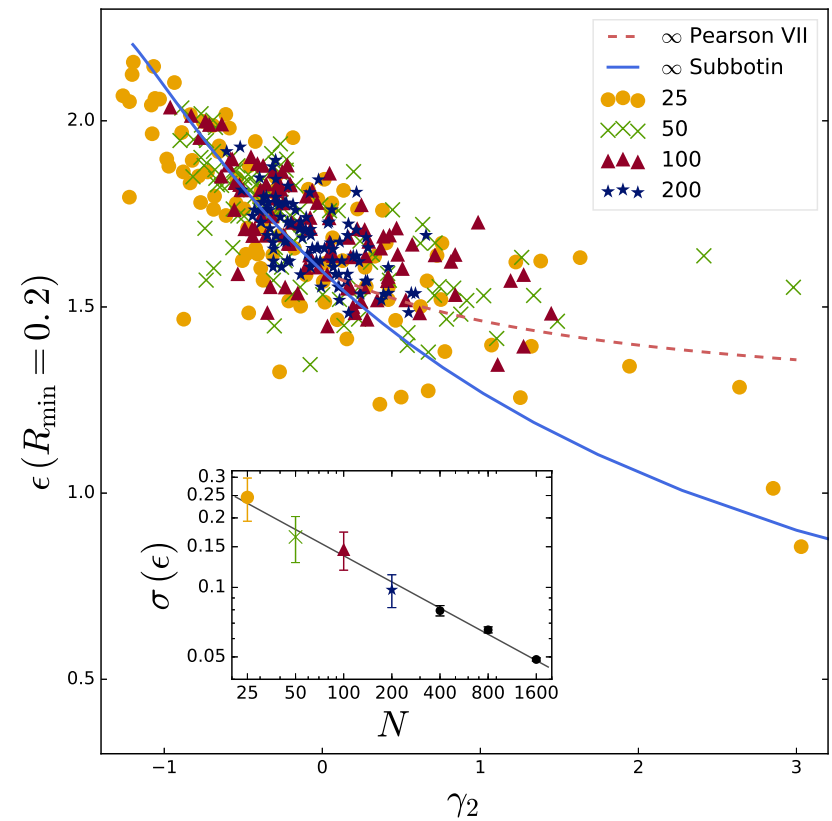

FIG. 4. (color online) Coupling strength at the level $R_{\min }=$ 0.2 vs. sample kurtosis of the respective $g(\omega)$-sample; the same data as in Fig. 3 are used. Solid and dashed lines give predictions from numerical integration of Eq. (4) for two families of distributions with kurtosis as a parameter (cf. Sec. VA . Inset: Scaling of the standard deviation of $\epsilon$ corresponding to $R_{\min }=0.2$ with the number of oscillators $N$; linearly fitted by power law $\sigma\left[\epsilon\left(R_{\min }=0.2\right)\right] \approx N^{-0.38}$.

Figure 3 shows that indeed the course and position of the curve $R_{\min }$ vs. $\epsilon$ is highly correlated with the sample kurtosis. One can differentiate three groups according to the darkness (color) of the curves: light-gray (pink) for the most negative observed excess kurtosis, gray (green) for samples with nearly vanishing $\gamma_{2}$, and black for the largest observed excess kurtosis. These three groups are well distinguishable for all ensemble sizes, and are characterized by the following properties:

1. Platykurtic samples of $g(\omega)$ (light gray, negative excess kurtosis) lift off from $R_{\text {min }}=0$ only at strong critical couplings. The curve climbs rapidly or even jumps to significant values of $R_{\min } \approx 0.4 \ldots 0.6$. These samples achieve full frequency synchronization at the lowest coupling strengths compared to other samples.

2. For leptokurtic samples (black, positive excess kurtosis), in contrast, comparably weak values of coupling suffice to synchronize a considerable central cluster. Thereby, $R_{\min }$ grows sedately from a rather small $\epsilon_{c}^{\mathrm{min}}$. Due to the constraint of unit variance, the outlier frequencies must be quite extreme and thus are eventually synchronized only by much stronger coupling. Consequently, these samples require stronger coupling to achieve full frequency locking.
3. Mesokurtic samples (small kurtosis, gray) naturally lie between these two extremes: they demonstrate roughly a "standard" transition in the curve $R_{\min }(\epsilon)$, similar to that demonstrated by the regular sample generated by virtue of quantiles (black dashed curves).

In Fig. 4 we quantify some of the qualitative observations above. Here we show the dependence of the value of the coupling parameter $\epsilon$ at which $R_{\text {min }}$ first crosses the threshold $R_{\min }=0.2$ on the excess kurtosis for different system sizes $N$. The data confirm the inverse proportionality mentioned above. As a theoretical substantiation, we compare the results from these numerical experiments in finite ensembles with the kurtosis dependence of $\epsilon\left(R_{\min }=0.2\right)$ in the thermodynamic limit; see Sec. V

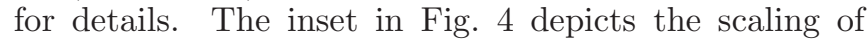
the standard deviation of $\epsilon$ at $R=0.2$ with system size $N$. As expected, the variability decreases in the limit $N \rightarrow \infty$ - approximately with $\Delta \epsilon \sim N^{-0.38}$.

\section{B. Sample skewness of $g(\omega)$ determines the drift of the global phase}

Random sampling of $g(\omega)$ results not only in variations of the shape of the distribution, characterized above by kurtosis but also in deviations from symmetry with respect to the mean value (the underlying Gaussian distribution is symmetric). These can be characterized by sample skewness $\gamma_{1}=\left\langle\omega_{i}^{3}\right\rangle\left\langle\omega_{i}^{2}\right\rangle^{-3 / 2}$. The main effect of skewness is a finite macroscopic frequency, $\Omega=\langle\dot{\varphi}\rangle_{t}$. Above, when discussing the theory in the thermodynamic limit, we argued that the macroscopic frequency vanishes for symmetric distributions. Figure 1(b) confirms that regular sampling via quantiles generates perfectly symmetric sets (for all $i$ there exists exactly one $j$ such that $\left.\omega_{i}=-\omega_{j}\right)$ and the global phase truly converges in the supercritical regime.

In Fig. 5, we present a numerical study on the dependence of the mean global frequency $\Omega$ on the skewness of $g(\omega)$ samples and on the coupling strength. First, we generate many Gaussian frequency samples and pick those with skewness close $\left( \pm 10^{-5}\right)$ to 1 of 20 target values of skewness, until having 20 samples for each. Each dot in the figure corresponds to the mean over the global phase velocities of the 20 samples with the same skewness. As explained above, $\Omega$ is only meaningfully defined for finite $R_{\text {min }}$, thus cases with $R_{\min }<0.1$ were rejected and the curves start from finite values of $\epsilon$. The individual global phases velocities are the slopes of linear fits of the global phase after cutting a transient of $10^{2}$ time steps.

To clarify the dependence of the observed macroscopic frequency on the ensemble size, we perform calculations similar to those presented in Fig. 5 for different values of $N$. Figure 6 displays how the correlation of the global angular velocity with sample skewness scales with ensemble size at a fixed coupling strength $\epsilon=2$. We generate 1000 


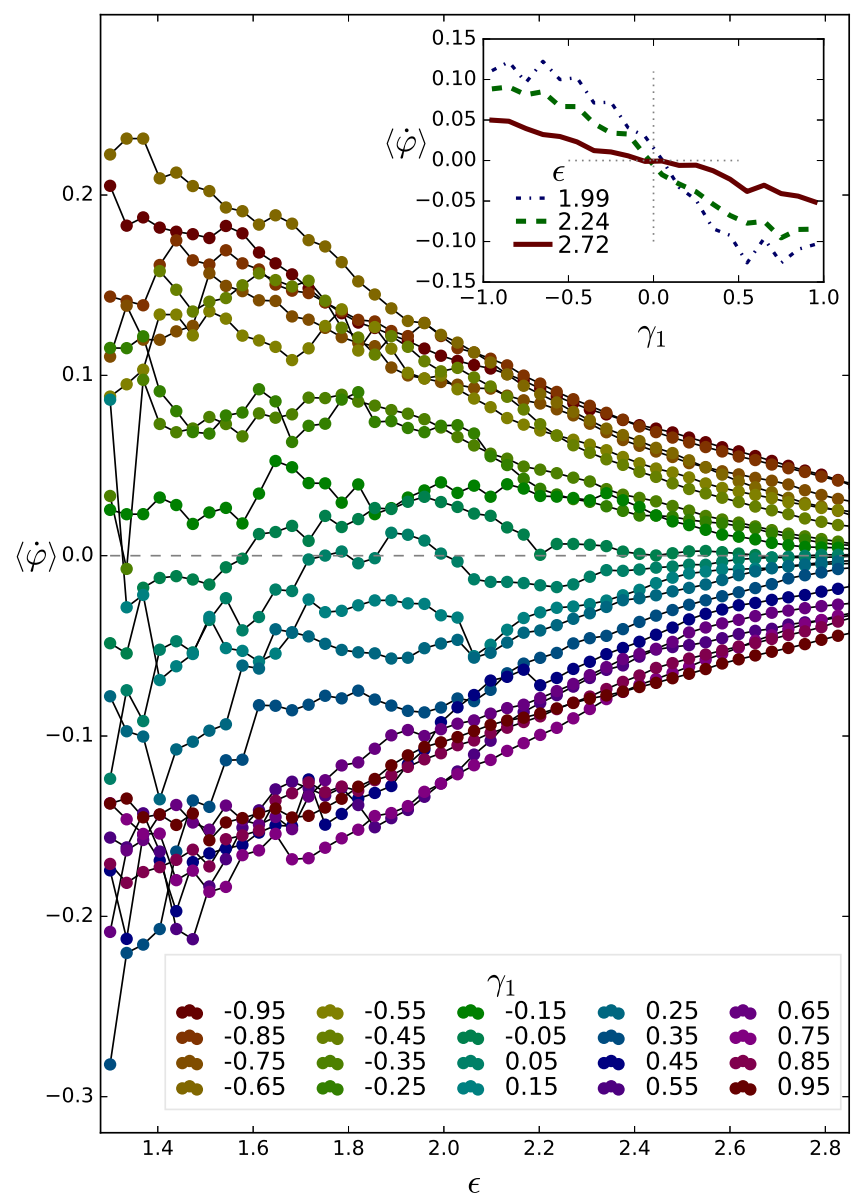

FIG. 5. (color online) Mean frequency of the macroscopic phase $\Omega=\langle\dot{\varphi}\rangle$ for $N=50$ oscillators versus coupling strength $\epsilon$. Different gray scales (colors in online version) indicate different sample skewnesses from top, $\gamma_{1}=-0.95$, to bottom, $\gamma_{1}=0.95$. Each chain of dots represents the mean over up to 20 phase velocities of distribution samples with the same $\left( \pm 10^{-5}\right)$ skewness (the relative number of samples with $R_{\min }>0.1$, i.e., for which a collective mode is actually defined at a given $\epsilon$, increases with coupling strength). The frequencies are calculated by averaging over the time interval $10^{3}$. The inset shows cuts through the main picture at three different values of $\epsilon$ : phase velocity vs. skewness.

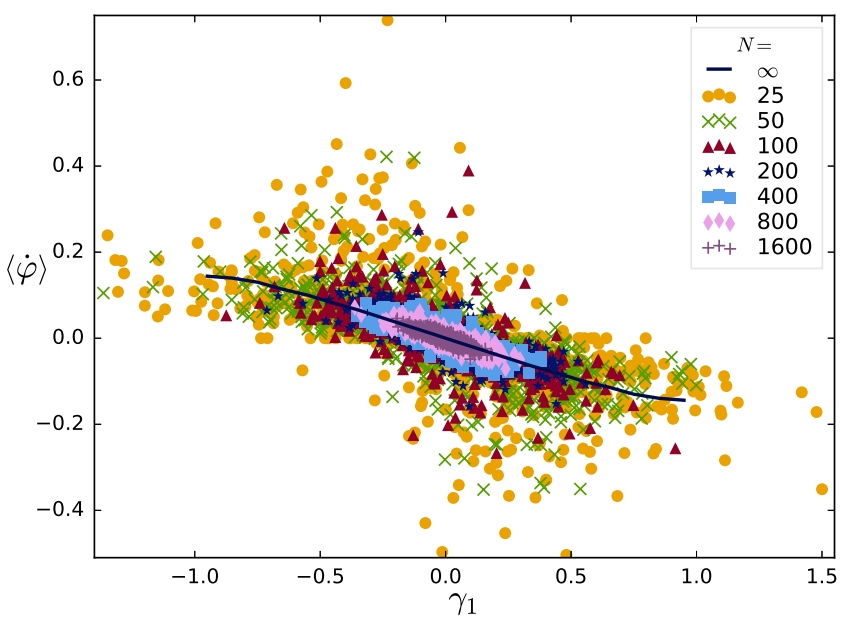

FIG. 6. (color online) Average frequencies of the mean field vs. skewness of the frequency sample for different ensemble sizes. The continuous curve stems from the numerical integration of self-consistency [Eq. (4)] for skew-normal distributions with different skewness parameters $\alpha$. Each marker shape corresponds to one ensemble size, for each of which 1000 samples of a Gaussian with mean zero and variance one are generated randomly. For each sample, the time evolution of $\varphi$ at $\epsilon=2$, performed over $t=10^{3}$ plus $10^{3}$ transient, gives $\langle\dot{\varphi}\rangle$ represented by one point in the plot.

\section{EFFECTS OF KURTOSIS AND SKEWNESS IN THE THERMODYNAMIC LIMIT}

In Sec. IV we demonstrated that a significant part of the variability of the parameter $R_{\min }$ and of the frequency of the collective mode $\Omega$ results from the sample variability of kurtosis and skewness, respectively. We further support these two findings by complementing them with calculations in the thermodynamic limit. In infinite ensembles, a full analysis of stationary solutions can be performed on the basis of Eq. (4), as explained in Sec. IIA above. Instead of using a symmetric standard distribution of frequencies, e.g., a Gaussian, we explore distributions with kurtosis and skewness as explicit parameters. We thereby also embed findings of Refs. 25, 38] for rather artificial distributions into the context of finitesize effects.

\section{A. Effect of kurtosis}

We start with kurtosis. One popular family of distributions, where kurtosis is a parameter, is the Pearson type VII family [39], but here excess kurtosis $\gamma_{2}$ ranges only from zero to infinity. More convenient for our purposes is the Subbotin family [40] (sometimes also called exponential power distribution), with excess kurtosis ranging from -1.2 to $\infty$. This family covers such important cases as uniform, Laplace, and Gaussian distributions.

The Subbotin family's probability density function dewith different $\gamma_{1}$, which we derive in Sec. $\mathbf{V}$

samples per $N \in[25,50,100,200,400,800,1600]$. In contrast to the former experiment, samples with all values of skewness enter the simulations. For small sample sizes, the spread in $\Omega$ for a fixed skewness is maximal. With increasing $N$, samples with high skewness get less likely. For $N=800,1600$, the form of dependence of $\Omega$ vs. $\gamma_{1}$ coincides very well with the theoretical curve obtained in the thermodynamic limit for skew-normal distributions 
pends on a main parameter $p$ (and on an auxiliary quantity $\sigma_{p}$ that serves for setting variance $\sigma$ to 1 ):

$$
g(\omega ; p)=\left[2 \sigma_{p} p^{1 / p} \Gamma(1+1 / p)\right]^{-1} \cdot \exp \left(-\frac{|\omega|^{p}}{p \sigma_{p}^{p}}\right),
$$

where $\Gamma(x)$ denotes the Gamma function. Notice the symmetry of the distribution for any $p$. The shape parameter $p$ acts inversely to the excess kurtosis $\gamma_{2}$; the exact relation is $\left.\gamma_{2}=\Gamma(1 / p) \Gamma(5 / p) /[\Gamma(3 / p))\right]^{2}$. The limit $p \rightarrow 0$, where $\gamma_{2} \rightarrow \infty$, corresponds to a delta distribution. The Laplacian distribution has $p=1$ and $\gamma_{2}=3$; it has a peak at zero and fat tails, comparable to a Lorentz distribution. With $p=2$, we have a Gaussian distribution with vanishing excess kurtosis. Finally, the case $p \rightarrow \infty$ yields a uniform distribution with excess kurtosis $\gamma_{2}=-1.2$.

The dependencies $R(\epsilon)$ for different values of the parameter $p$ can be obtained from Eq. (41). Because the imaginary part of the integral vanishes due to symmetry, we only have to calculate the first real integral in Eq. (4), which reduces to

$$
R=\int_{-a}^{a} g(\omega ; p) \sqrt{1-(\omega / a)^{2}} \mathrm{~d} \omega, \quad \epsilon=a / R,
$$

by transforming to $\omega=a \sin \theta$. This expression clearly shows the role of the distribution of the probability mass to either the center or the tails of the distribution $g(\omega ; p)$ : The tails beyond $|\omega|=a$ do not contribute to the integral.

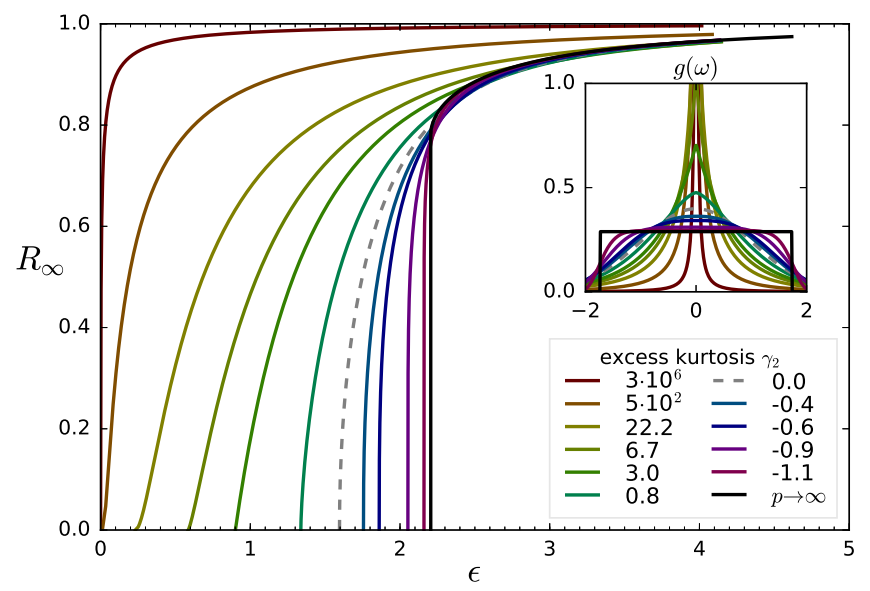

FIG. 7. (color online) Kurtosis dependence of $R(\epsilon)$ : We numerically integrate the self-consistent Eq. (9) for the Subbotin family of frequency distributions, see Eq. (8). The integral for the uniform distribution stems from the analytical solution, see Eq. (77) with $\omega_{\max }=\sqrt{3}$. The curves (from left to right) correspond to values of the parameter $p=0.1,0.25,0.5,0.75,1,1.5,2,2.5,3,5,10$ and to the uniform distribution with $p \rightarrow \infty$ (or likewise to the excess kurtosis values as given in the legend). The inset shows the respective probability densities.
We discussed the analytical integration of some of representatives $g(\omega ; p)$ in the end of Sec. IIA For all other values of $p$, we solve the integral in Eq. (9) numerically. Figure 7 shows numerical solutions for different $p$ and thus for different excess kurtosis values $\gamma_{2}$, as well as the analytical solution for $p \rightarrow \infty$, corresponding to a uniform distribution. The $R(\epsilon)$ curves stemming from the Subbotin family qualitatively fit numerical results of Fig. [3 For a quantitative correspondence, it is sufficient to remind that the critical value of the coupling constant $\epsilon_{c}$ is inversely proportional to $g(0 ; p)$, which in turn grows with kurtosis. Thus one obtains inverse proportionality

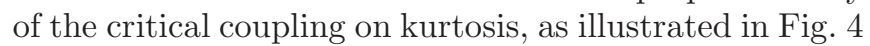
via the dashed curve for the Pearson VII family, and via the solid curve for the Subbotin family.

\section{B. Effect of skewness}

Next, we discuss the connection between skewness and the mean frequency of the macroscopic oscillations by exploring a family of skewed normal distributions

$$
g(\omega ; \alpha)=\frac{1}{\sqrt{2 \pi} \sigma_{\alpha}} \mathrm{e}^{-\omega^{2} / 2 \sigma_{\alpha}}\left[1+\operatorname{erf}\left(\frac{\alpha \omega}{\sqrt{2} \sigma_{\alpha}}\right)\right]
$$

where erf is the error function and the parameter $\alpha$ sets the skewness $\gamma_{1}=\frac{4-\pi}{2} \alpha^{3} \cdot\left[\frac{\pi}{2}\left(1+\alpha^{2}\right)-\alpha^{2}\right]^{-3 / 2}$.

Numerical solution of Eq. (4) for the asymmetric case requires an additional step, namely finding the value of $\Omega_{a}$ for each $a$, such that the imaginary part of the integral in Eq. (4) vanishes. We first simplify the asynchronous integral for a general skewed distribution ( $\alpha$ fixed) to

$$
\begin{aligned}
\mathcal{I}_{\text {asy }}= & \frac{i}{a}\left[\int_{a}^{\infty} \mathrm{d} \omega g(\omega+\Omega)\left(2 \omega-\sqrt{\omega^{2}-a^{2}}\right)\right. \\
& \left.+\int_{-\infty}^{-a} \mathrm{~d} \omega g(\omega+\Omega)\left(2 \omega+\sqrt{\omega^{2}-a^{2}}\right)\right] \stackrel{!}{=} 0 .
\end{aligned}
$$

Note that this integral explicitly balances out the tails of $g(\omega)$ starting from $a$, i.e., asymmetries inside of $[-a, a]$ have no effect on the global phase velocity. We iteratively approach $\Omega_{a}$ for each $a$ using the Newton-Raphson method. As mentioned before, $\Omega_{a}$ is unique for each $a$, but still in order not to miss any roots, we explored a range $-4<\Omega<4$ - far beyond typical frequencies in $g(\omega ; \alpha)$. Figure 8 shows the results; we use the same skewnesses as in Fig. 5 for a straightforward comparison. The correspondence to the numerical experiment with finite $N$ is apparent for large values of the coupling parameter, while close to the transition, where averaging in Fig. 5 covers less than 20 distributions, deviations are large. At a fixed value of coupling $\epsilon=2$, the dependence of frequency $\Omega$ on the skewness in the family Eq. (10) is shown with a solid line in Fig. 6. This dependence fits the numerical data very well for finite large ensembles. 


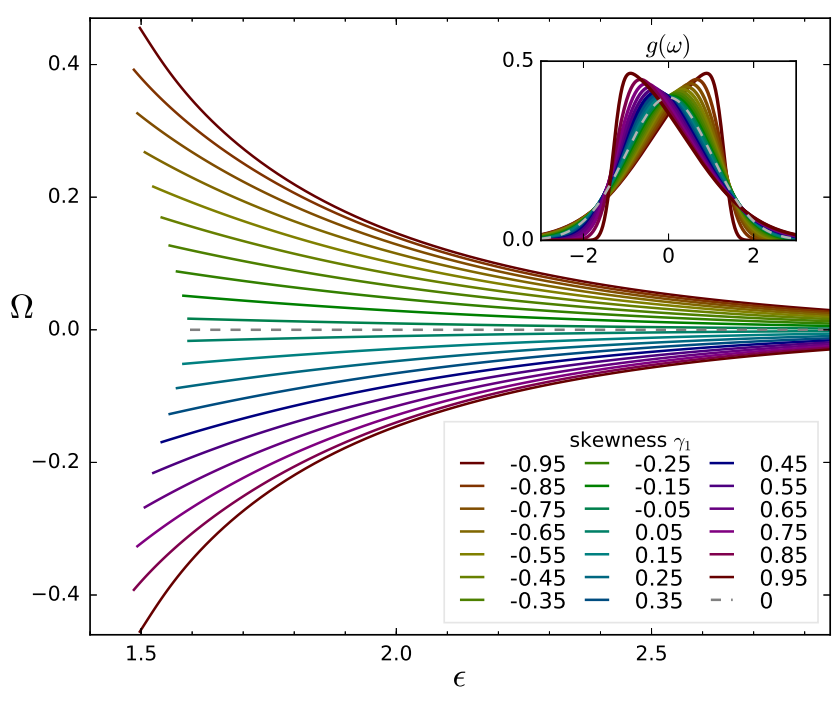

FIG. 8. (color online) Frequency of the macroscopic oscillations $\Omega$ for skewed normal distributions as in Eq. (10), obtained via solving the self-consistency relation Eq. (11) for skewness from top $\gamma_{1}=-0.95$ to bottom $\gamma_{1}=0.95$. The distributions are depicted in the inset.

\section{CONCLUSIONS}

Motivated by the approach of Ref. [17], we characterize the transition to synchrony in finite populations of phase oscillators as the emergence of a well-defined collective mode. A collective phase is well defined only if a collective amplitude never vanishes. Therefore, the minimum of the Kuramoto order parameter over a sufficiently long observation time, $R_{\min }$, serves as a proper criterion for the presence of a collective mode by ensuring a meaningful definition of the Kuramoto global phase at all times. In contrast to the time-averaged value of the order parameter, $R_{\min }$ undergoes a sharp transition. This allows us to determine a critical coupling strength $\epsilon_{c}$ for the synchronization transition in each sample. This transition point can be determined with high precision by extending the time series along which the minimum is retrieved.

Furthermore, we attribute the variations of the coupling dependence of the explored parameter $R_{\min }$ to the variations of the effective shape of the underlying frequency distribution, measured by kurtosis. Under the constraint of unit variance, kurtosis of a finite frequency sample allows us to distinguish whether either most frequencies are crowded close to the mean and few extreme outliers balance the variance or all frequencies are rather broadly distributed but without extremes. All samples lie between these two limiting cases of platykurtic and leptokurtic samples, respectively. In leptokurtic samples, the central frequencies synchronize at comparably low coupling strength and their critical coupling is accordingly small. The few extreme outliers require much stronger coupling to adjust their frequency to the central cluster. In contrast, platykurtic samples have comparably large values of critical coupling $\epsilon_{c}$ but then rapidly climb or even jump to high values of the order parameter, eventually reaching full frequency locking at lower coupling strengths compared to platykurtic samples. These properties are well reproduced in the thermodynamic limit, shown by numerically calculating the stationary solution for the order parameter for a family of distributions with selectable kurtosis.

The second observation in finite ensembles where we were able to treat the thermodynamic limit in a similar way is the dependence of the mean frequency of the global phase on the sample skewness of some natural frequency distribution. The mean frequency and skewness are roughly anticorrelated. Ensembles with perfectly symmetric frequency samples converge to a constant, nonrotating phase at any coupling strength above transition.

Note that the considerations regarding the effects of kurtosis and skewness on the dynamics in the thermodynamic limit cannot straightforwardly be extended to distributions that do not possess the corresponding moments. A prominent example due to its analytical tractability is the Lorentz distribution. Actually, the only representative of the important family of alphastable distributions with defined moments other than the mean is the Gaussian distribution. Though moments are undefined, this distribution family has parameters for asymmetry and shape.

The approach of Sec. \A for the thermodynamic limit can be applied to distributions of any asymmetry and shape, provided the integrals in Eq. (44) converge. A remaining challenge lies in finding a meaningful procedure to relate sample asymmetry and sample shape for finite samples of the Lorentz distribution to families of continuous distributions, as we presented for the Gaussian ensemble in Sec. V

Conceptually, a fat-tailed distribution of frequencies potentially contradicts the model assumption of nearly identical oscillators. In finite samples, the effect of extreme outliers on the collective dynamics is, however, comparably small due to a strong separation of time scales. The usage of the value $R_{\min }$ as an indicator for the transition is therefore applicable to the Lorentz ensemble and to other fat-tailed distributions.

In this paper we applied the criterion of the existence of a global oscillatory mode, based on the existence of the global phase, to the Kuramoto model. It would be interesting to explore finite-size effects on collective modes also for other types of synchronization transitions, where, e.g., all oscillators remain unlocked and the collective mode is related to partial synchrony, see Refs. [41, 42]. 


\section{ACKNOWLEDGMENTS}

This paper was developed within the scope of the IRTG 1740/TRP 2015/50122-0, funded by the DFG/ FAPESP. In studies presented in Sec.V. A.P. was supported by the Russian Science Foundation (Grant No. 17-12-01534). We thank Ralf Toenjes for valuable discussions.

[1] M. Nixon, E. Ronen, A. A. Friesem, and N. Davidson, Phys. Rev. Lett. 110, 184102 (2013).

[2] I. Z. Kiss, Y. Zhai, and J. L. Hudson, Science (N.Y.) 296, 1676 (2002).

[3] A. E. Motter, S. A. Myers, M. Anghel, and T. Nishikawa, Nat. Phys. 9, 191 (2013).

[4] E. G. Antzoulatos and E. K. Miller, Neuron 83, 216.

[5] A. T. Winfree, The Geometry of Biological Time, 1st ed. (Springer, Berlin,, 1980).

[6] Y. Kuramoto, Math. Probl. Theor. Phys. 39, 420 (1975).

[7] Y. Kuramoto, Chemical Oscillations, Waves, and Turbulence, Springer Series in Synergetics, Vol. 19 (Springer, Berlin, 1984).

[8] H. Daido, Phys. Rev. Lett. 77, 1406 (1996)

[9] M. Komarov and A. Pikovsky, Physica D: Nonlinear Phenomena 289, 18 (2014).

[10] H. Sakaguchi and Y. Kuramoto, Progress of Theoretical Physics 76, 576 (1986).

[11] J. A. Acebrón, L. L. Bonilla, C. J. P. Vicente, F. Ritort, and R. Spigler, Reviews of Modern Physics 77, 137 (2005).

[12] A. Arenas, J. Kurths, Y. Moreno, A. Díaz-Guilera, and C. Zhou, Physics Reports 469, 1 (2008)

[13] S. Watanabe and S. H. Strogatz, Physica D: Nonlinear Phenomena 74, 197 (1994).

[14] E. Ott and T. M. Antonsen, Chaos 18 (2008), 10.1063/1.2930766NoStop

[15] A. Prindle, P. Samayoa, I. Razinkov, T. Danino, L. S. Tsimring, and J. Hasty, Nature 481, 39 (2012)

[16] A. Weber, Y. Prokazov, W. Zuschratter, and M. J. B. Hauser, PLoS ONE 7, 1 (2012)

[17] A. A. Temirbayev, Z. Z. Zhanabaev, S. B. Tarasov, V. I. Ponomarenko, and M. Rosenblum, Phys. Rev. E 85, 015204 (2012).

[18] O. V. Popovych, Y. L. Maistrenko, and P. A. Tass, Phys. Rev. E 71, 065201 (2005).

[19] H. Daido, Journal of Physics A: Mathematical and General 20, L629 (1987).

[20] H. Daido, Journal of Statistical Physics 60, 753 (1990)

[21] S. W. Son and H. Hong, Physical Review E - Statistical, Nonlinear, and Soft Matter Physics 81, 1 (2010).

[22] H. Hong, H. Chaté, H. Park, and L. H. Tang, Phys. Rev. Lett 99, 1 (2007), arXiv:0701646

[23] H. Hong, H. Chaté, L. H. Tang, and H. Park, Physical Review E - Statistical, Nonlinear, and Soft Matter Physics 92, 1 (2015).
[24] L. Basnarkov and V. Urumov, Physical Review E - Statistical, Nonlinear, and Soft Matter Physics 76, 1 (2007).

[25] D. Pazó, Physical Review E - Statistical, Nonlinear, and Soft Matter Physics 72, 1 (2005).

[26] D. Pazó and E. Montbrió, Phys. Rev. E 80, 046215 (2009).

[27] O. E. Omel'chenko and M. Wolfrum, Phys. Rev. Lett. 109, 164101 (2012).

[28] O. E. Omel'chenko and M. Wolfrum, Physica D 263, 74 (2013).

[29] X. Zhang, A. Pikovsky, and Z. Liu, Nat. Sci. Rep. 7 (2017).

[30] M. Abramowitz and I. Stegun, Handbook of Mathematical Functions (Dover, London,, 1965).

[31] H. Hong, H. Park, and L. H. Tang, Physical Review E - Statistical, Nonlinear, and Soft Matter Physics 76, 1 (2007), arXiv:0710.1137

[32] J. C. Bronski, L. DeVille, and M. J. Park, Chaos 22, 033133 (2012).

[33] H. Chiba and I. Nishikawa, Chaos 21, 043103 (2011)

[34] H. Dietert, J. Math. Pur. Appl. 105, 451 (2016).

[35] C. Choi, M. Ha, and B. Kahng, Phys. Rev E. 88, 032126 (2013).

[36] The authors of Ref. [43] refine this simplified description to "kurtosis vaguely [is the] location- and scale-free movement of probability mass from the shoulders of a distribution into its center and tail."

[37] Generating a sample from a Gaussian with two or more clearly distinct modes (maxima) is rather improbable, we do not discuss them here. We just notice that already bimodal frequency distributions exhibit a rich bifurcation map [26, 44].

[38] S. Petkoski, D. Iatsenko, L. Basnarkov, and A. Stefanovska, Phys. Rev. E 87, 032908 (2013).

[39] K. Pearson, Philosophical Transactions of the Royal Society of London A: Mathematical, Physical and Engineering Sciences 216, 429 (1916).

[40] M. Subbotin, Matematicheskii Sbornik 31, 296 (1923)

[41] C. van Vreeswijk, Phys. Rev. E 54, 5522 (1996)

[42] P. Clusella, A. Politi, and M. Rosenblum, New Journal of Physics 18, 093037 (2016).

[43] K. P. Balanda and H. L. MacGillivray, Am. Stat. 42, 111 (1988).

[44] E. A. Martens, E. Barreto, S. H. Strogatz, E. Ott, P. So, and T. M. Antonsen, Phys. Rev. E 79, 026204 (2009). 